\title{
Vulval lymphangiectasia
}

\author{
S E HANDFIELD-JONES, W J PRENDIVILLE, S NORMAN \\ From the Vulva Clinic, Departments of Dermatology, Gynaecology, and Venerology, Bristol Royal Infirmary, \\ Bristol, UK
}

SUMMARY Three cases of lymphangiectasia of the vulva are reported. One case followed Wertheim's hysterectomy for carcinoma of the cervix; two other cases had Crohn's disease with perineal involvement. Lymphangiectasia is a secondary phenomenon resulting from obstruction of previously normal lymphatics. This is in contrast with lymphangioma which is an anatomical abnormality. A review of the literature suggests that the vulval skin may be particularly susceptible to the formation of lymphangiectasia, and our patients' experiences suggest that they are easily mis-diagnosed.

\section{Case reports}

\section{Case 1}

This 38 year old woman presented to us in 1986, complaining of lumps on the vulval area, which discharged clear fluid. She had had a Wertheim's hysterectomy in 1977, for invasive carcinoma of the cervix, followed by radiotherapy; total dosage 5209 cGy. She developed minor swelling of the thighs shortly afterwards, and had several episodes of cellulitis of the thighs and suprapubic area. In 1984 she first complained of leakage of fluid from the vulva, and received anti-candidal therapy without a clear diagnosis being made.

When first seen by us she had minor swelling of the right labia, and multiple shiny "frogspawn" like vesicles on the right and left labia, which exuded clear fluid when punctured (fig 1). A biopsy was performed which showed enlarged superficial lymphatic channels with endothelial lining. Overlying epidermis showed hyperkeratosis. Despite warnings of likely recurrence, the patient wanted any therapy available. A superficial skinning laser vulvectomy to 2-3 mms was performed in 1987. She was free of problems at two months, but unfortunately failed to attend for further follow up.

\section{Case 2}

This patient developed Crohn's disease at the age of 21 years, and had a total colectomy. From 1974 to 1976 she had multiple perineal fistulae, and these were laid open and healed. In 1983 she first noticed weeping

Address for reprints: Dr S Handfield-Jones, 8 Doris Road, Norwich NR2 3EJ, UK.

Accepted for publication 30 June 1989

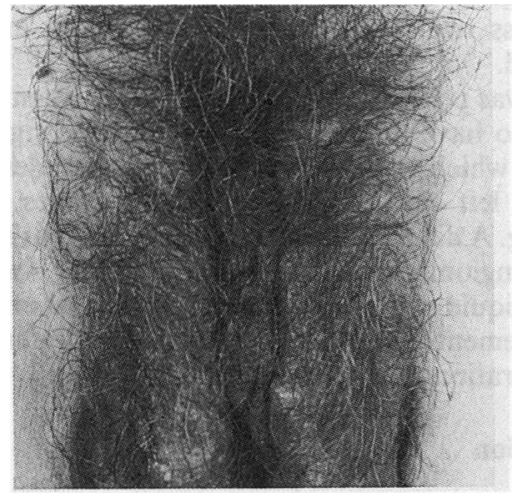

Fig 1 Case 1 showing vesicles on labia majora.

lumps on the vulva. She was treated for candida, and investigated for herpes simplex infection and folliculitis. She was first seen by us in 1985, when she was aged 38 years, and was noted to have vesicles on both labia minora without obvious lymphoedema. She had multiple scars from previous fistulae, but no open or active fistulae. Histology showed cystically dilated lymph vessels lined by a single layer of endothelial cells.

Local resection of the most severely affected areas was performed in 1986, and although not clear of the condition the patient has remained comfortable.

\section{Case 3}

This woman, born in 1949, developed Crohn's disease in her teens. She had a local bowel resection and later developed several perianal fistulae which were laid open. In 1984, when her Crohn's disease was clinically 


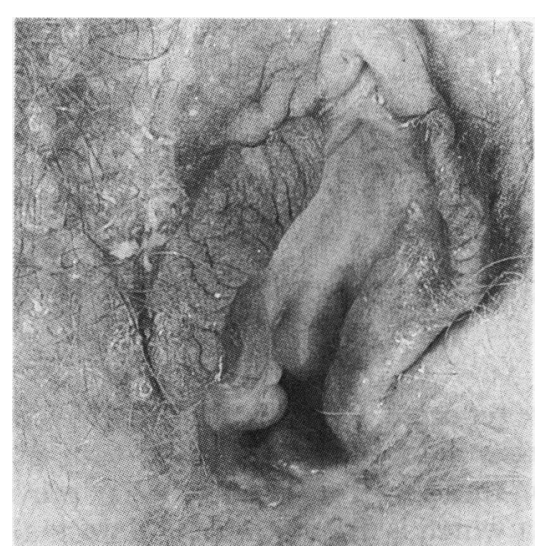

Fig 2 Case 2, vesicles and hyperkeratosis on right labia.

quiescent, she first noticed vulval lumps. These were diagnosed as viral warts. Cryotherapy resulted in discharge of clear fluid, and once healed the lesions were less troublesome for several weeks, and then relapsed.

She was referred to our clinic in 1986 , when she was found to have mild lymphoedema of the right labia majora which was covered with clear papules (fig 2). On the left side there were a few papules, but no swelling. A diagnostic biopsy was reported as showing lymphangioma circumscriptum. Further treatment with liquid nitrogen resulted in short term improvement, but she has since been referred for consideration of surgical treatment.

\section{Discussion}

Abnormal dilation of the lymphatics of the skin can be either a primary structural abnormality known as lymphangioma, or a secondary event following lymphatic obstruction, known as lymphangiectasia. Lymphangiomas have been classified by Peachey ${ }^{1}$ into classicial lymphangioma circumscriptum and localised lesions. The aetiology is believed to be an isolated but often deep developmental abnormality of the lymphatics. Lymphangiograms have shown the abnormal lymphatics to be totally separate from the main lymphatic drainage in some cases. ${ }^{2}$

The aetiology and terminology of acquired lymphangiectasia is less well defined. A wide variety of inflammatory, neoplastic or surgical insults can block lymphatics and the usual outcome is lymphoedema. ${ }^{3}$ The skin in advanced lymphoedema often becomes thickened and warty and may show lymphangiectases. ${ }^{3}$ The eventual result can be malignant change as in the post mastectomy lymphangiosarcoma of Stewart and Treves. ${ }^{4}$

Our cases are unusual in that they show lymphan- giectasia, without marked or tense lymphoedema. Previously published cases of lymphangiectasia with minor oedema appear to be secondary to local dermal lymphatic obstruction, such as keloid scars $^{5}$ and scrofuloderma,${ }^{6}$ rather than proximal obstruction at the draining nodes.

Genital skin affected with lymphangiectasia has been described before with both scrotal lesions ${ }^{78}$ and vulva lesions. Three previous cases of lymphangiectasia of the vulva have been reported following carcinoma of the cervix. One patient was treated with radiotherapy alone, ${ }^{9}$ but was complicated by episodes of cellulitis. The second had radiotherapy and extraperitoneal pelvic lymph node clearance, and also suffered from recurrent cellulitis of the thighs and perineum..$^{10}$ The third patient had a radical hysterectomy, and radiotherapy." "Whilst the latter two patients had lymphoedema of the thighs, none had marked vulval oedema. All developed weeping vesicles which were very similar to those seen in our patients. A quite distinct aetiology was described by Heuvel, ${ }^{12}$ who reported vulval lymphangiectases secondary to lymph node tuberculosis. This patient did have marked oedema of the vulva, and lymphatic obstruction was compounded by multiple surgical procedures to drain suppurating inguinal lymph nodes.

Crohn's disease has not, to our knowledge, been reported as a cause of vulval lymphangiectasia. Vulval involvement in Crohn's disease is well recognised ${ }^{1314}$ with granulomatous lesions both contiguous with perianal disease and as a metastatic process, and this may present as lymphoedema. Although our patients with Crohn's disease had had fistulation of the perineum this was inactive when we saw them, and there was no evidence of granulomatous Crohn's in the perianal skin. Treatment of the condition is extremely difficult. In lymphangioma circumscriptum it is stressed that the disease recurs unless the full depth of the abnormal lymphatics are removed. ${ }^{2}$ Secondary lymphangiectases are more of a problem as the obstruction is, presumably, diffuse and complete surgical clearance is likely to be impossible. Although laser vulvectomy was undertaken in one of our patients, we are not optimistic about the long term outcome.

In none of the patients reported by both ourselves and other authors, is the causative pathology rare or extreme. All three of our patients had been misdiagnosed for at least two years, and it is possible that there are many cases that are overlooked. The symptom of discharge of clear fluid and the sign of "frogspawnlike" vesicles should alert clinicians to the diagnosis.

We thank Dr J Burton for allowing us to present Case 2, and the Histopathology Departments at Bristol Royal Infirmary and the Bristol General Hospital. 


\section{References}

1 Peachey RDG, Lim CC, Whimster IW. Lymphangioma of Skin. Br J Dermatol 1970;83:519-27.

2 Whimster IW, The Pathology of lymphangioma circumscriptum. Br J Dermatol 1976;94:473-86.

3 Kinmonth JB. The lymphatics; surgery, lymphography and diseases of the chyle and lymph systems. Baltimore: E Arnold, 1982.

4 Stewart FW, Treves N. Lymphangiosarcoma in postmastectomy lymphoedema. Cancer 1948;1:64-81.

5 Russell B. Lymphangioma circumscriptum and keloids. $\mathrm{Br} J$ Dermatol 1951;63:158-9.

6 Di Leonardo M, Jacoby RA. Acquired cutaneous lymphangiectases secondary to scarring from scrofuloderma. J Am Acad Dermatol 1986;14:688-90.

7 Russell B, Pridie RB. Lymphoedema of scrotum. Br J Dermatol
1967:79:298.

8 Weakley DR, Juhlin EA. Lymphangiectases and lymphangiomata. Arch Derm Syph 1961;84:574-8.

9 Fisher I, Orkin M. Acquired lymphangioma (lymphangiectasis). Arch Dermatol 1970;102:230-4.

10 La Polla J, Foucar E, Leshin B, et al. Vulval lymphangioma circumscriptum; a rare complication of therapy for squamous cell carcinoma of the cervix. Gynecol Oncol 1985;987-9.

11 Young N. Lymphangioma of vulva. NY State J Med 1980:987-9.

12 Heuvel NVD, Stolz E, Notowicz A. Lymphangiectasias of the vulva in a patient with lymph node tuberculosis. Int J Dermatol 1979;18:65-6.

13 Lavery HA, Pinkerton JHM, Sloan J. Crohn's disease of the vulva - two further cases. Br J Dermatol 1985;113:359-63.

14 McCallum DI, Kinmont PDC. Dermatological manifestations of Crohn's disease. Br J Dermatol 1968;80:1-7. 